\title{
The Open Mathematics of Crystallization
}

Charles Radin

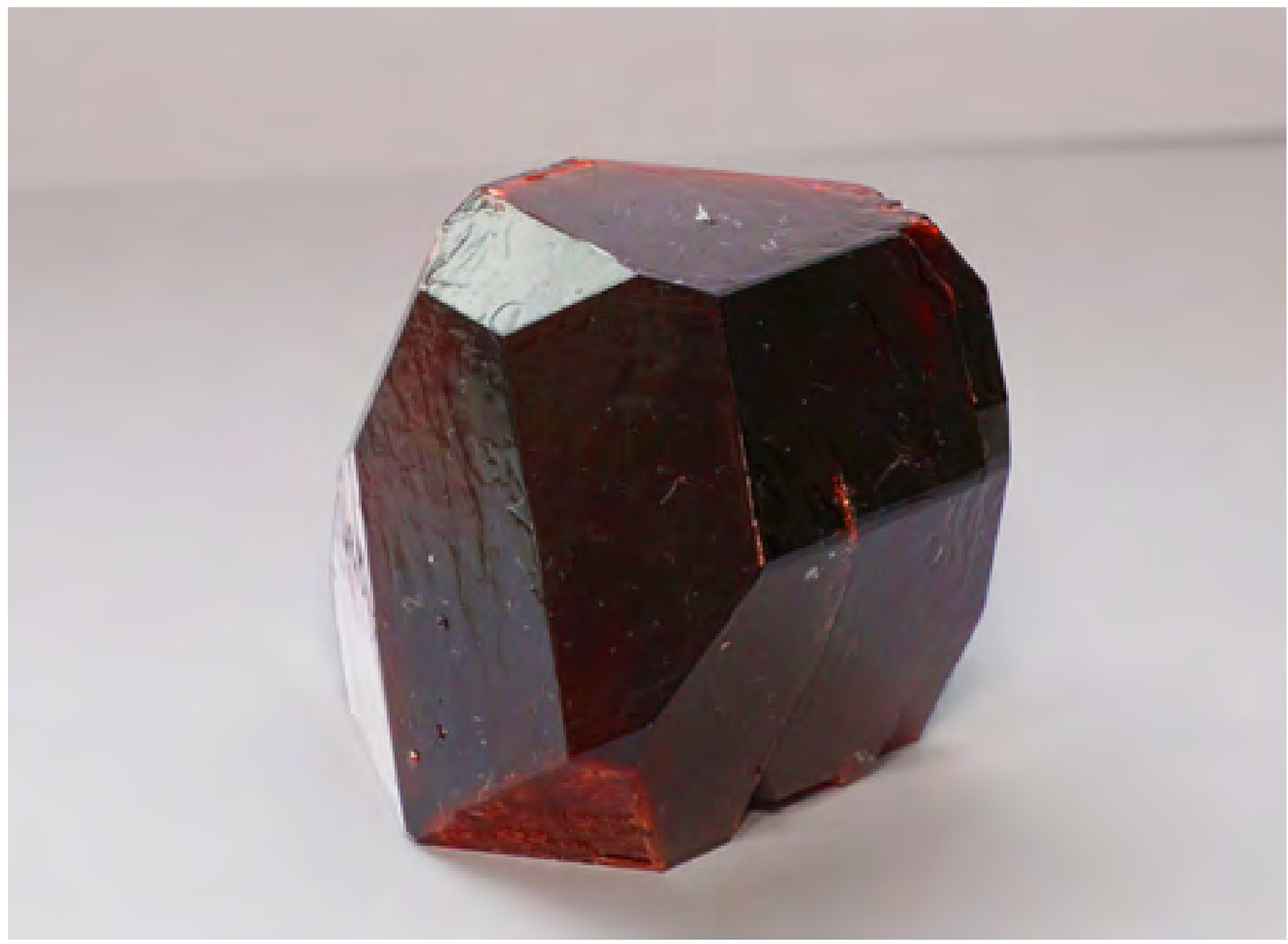

Ammonium-cobalt-sulfate crystal.

Abstract. We discuss open mathematical questions in modelling the transition from water to ice and such phenomena.

Charles Radin is professor of mathematics at the University of Texas at Austin. His e-mail address is radin@math. utexas . edu. This research was supported in part by NSF Grant DMS-1509088. For permission to reprint this article, please contact: reprint-permission@ams.org.

DOI: http://dx.doi.org/10.1090/noti1534
A drop of water suspended in a bath of oil is round because of the pull of its surface tension. The spherical shape is not a surprise because of the symmetry of all the forces on it. However, if the drop of water is then frozen into ice its surface becomes polyhedral, which suggests that somehow the symmetry decreases. The decrease is eloquent evidence of something not visible to the eye: the crystalline configuration of the water molecules in the water after it turns to ice (see Figure 1). Slightly different situations create facets in salt, quartz, and many other minerals, as in Figure 2. Our goal is to give an overview 


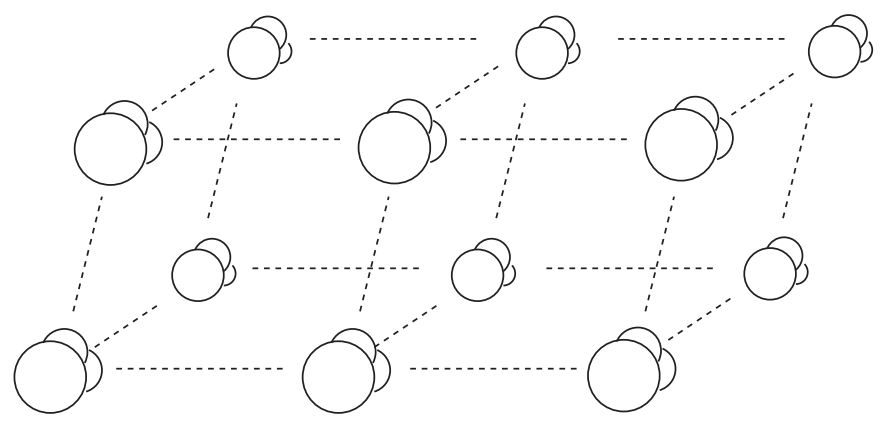

Figure 1. When water turns to ice it assumes a crystalline configuration.

of several open problems connected by a common origin of "minimization of surface tension," the mathematics coming from probability and the calculus of variations. The narrative will fork several times, the various directions leading to open problems, some of which are old and famous, and will use intuitive arguments where there are gaps in the mathematics.

The creation of polyhedral shapes for the boundary of crystalline collections of molecules is widely believed to follow as a straightforward exercise in statistical physics. Disclaimer: no one has actually been able to prove that crystalline configurations follow automatically from the standard statistical physics model at low but nonzero temperatures, let alone the minimal surface tension shape boundary which should then follow! Still, the difficulty of such proofs is believed to be "merely technical," and the basic structure of this theory for the study of bulk matter has been universally accepted for about one hundred years, during which our focus has gradually shifted to less familiar material phenomena such as superconductivity and superfluidity. In the midst of studying these more exotic behaviors, physicists became interested in various types of two-dimensional matter, for instance graphene, which is a sheet of carbon atoms bound together in a hexagonal lattice, sometimes just one atom thick. The 2016 Nobel Prize in Physics (see p.557) was awarded for developing topological methods to study exotic behavior in two-dimensional materials and in 2010 was given for experimental developments on graphene. This article will analyze the dusty old phenomena of crystallization and faceting, but for the more interesting two-dimensional matter. In two dimensions some basics are unknown, not only in the mathematical modelling but even in the experimental physics it should reproduce.

We begin with the more familiar three-dimensional situation before modifying for two dimensions.

First of all, by going to a model at the molecular level we will have to replace the macroscopic notion of surface tension, which makes sense as a (dissipative) force on the water drop as a whole but not as a force between individual water molecules. At the molecular level it is appropriate and traditional to reexpress the effect of surface tension in terms of the change in the energy of the water molecules due to the presence of their interface with the oil. We want to know how the so-called ideal "Wulff shape" for our

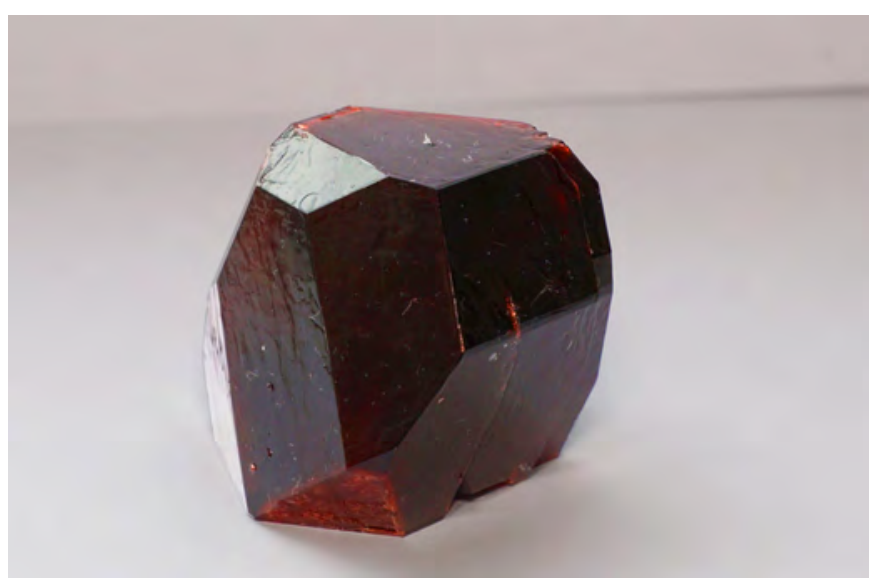

Figure 2. The ammonium-cobalt-sulfate crystal assumes a faceted polyhedral shape due to the underlying crystal structure.

water drop is thereby determined by the interacting water molecules, why that shape changes with temperature, and how that shape reveals the hidden molecular structure.

To give some context, we note that the basic analysis of the solid, liquid, and gas phases of matter, and of the transformations between such phases is part of thermodynamics developed in the nineteenth century. Thermodynamics focuses on energy and models how substances change when energy flows between materials due to a variety of possible processes, including heating/cooling. Scientists became increasingly convinced that the laws of thermodynamics should be straightforward consequences of the interactions of constituent particles (atoms), a prescient conviction since the atomic structure of matter was not convincingly demonstrated until much later. The new, deeper analysis of energy in materials is called (equilbrium) statistical mechanics, and we will discuss such an analysis for the freezing of two-dimensional matter, the polygonal shape of such frozen matter.

The formalism of statistical mechanics is surprisingly flexible and is easily adapted to particles in any spatial dimension, so we will start with the familiar dimension three. To understand through statistical mechanics why a water drop freezes and develops facets, one might reasonably start with the nuclei and electrons making up all the atoms in water. The energy of such a system of particles consists of their individual kinetic energies plus the potential energy associated with the forces of interaction; the only forces they can feel are the well-known electromagnetic forces associated with their charges. (Gravity can be ignored in the modelling, if not in experiment!) It turns out to be hopeless to analyze the freezing of matter in this way. Instead one normally uses a simplified, or toy, model of the material in which the nuclei and electrons are assumed to have "previously" formed molecules, which then interact through some artificial force law, which, to capture realistic influences, would consist of a strong repulsion at small separation and weak attraction out to some larger separation. For 
instance we could assume the force was infinite and repulsive for separation less than 1 and constant and attactive for separations between 1 and 3: hard balls that attract for some distance. More specifically, one would get such a force as the negative gradient of the following potential energy function of the separation $r$ between any particle pair:

$$
\phi(r)= \begin{cases}+\infty, & \text { if } r<1 \\ r-3, & \text { if } 1 \leq r \leq 3 \\ 0, & \text { if } r>3\end{cases}
$$

This is a reasonable approach for many materials of interest (though not for metals, among others), and for simplicity we use this traditional approach. It has the advantage that it removes any necessity for quantum mechanics, which disappears into the formation of the molecules. One thus imagines the material to be made of molecules modelled as small impenetrable unit volume balls which attract one another out to some small multiple of their radii. The fact then, which is at least coun-

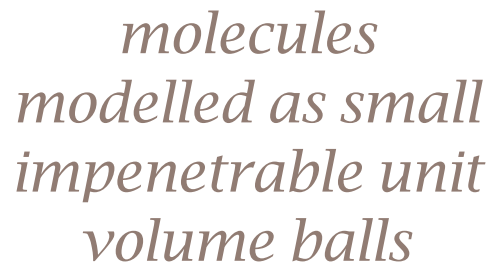

terintuitive if not mind boggling, is that a large collection of such interacting balls would automatically freeze into a crystalline configuration when energy is removed, i.e. when cooled, and do so at some infinitely precise temperature, which is $0^{\circ}$ Celsius for water!

We will define the formalism and sketch first how it is used to analyze a water drop in an oil bath and then the differences in two dimensions, our real focus. As its name suggests, statistical mechanics uses probabilistic tools, though from it we expect to get information about the polyhedral shape of a crystal as in Figure 2, which is as nonrandom as anything in nature. Technically, probability is used to model the precise state of $\approx 10^{23}$ molecules, and the randomness disappears when one considers macroscopic quantities such as the shape of a chunk of ice.

Here is the framework. Imagine a large number $N$ of point particles, the centers of the molecules, in a cubic box $B_{V}$ of volume $V$ in $d$-dimensional space, $d=2$ or 3, and interacting via (1). The 'state' of the $j$-th particle consists of its position $x_{j}$ in $B_{V}$ and its velocity $v_{j}$ in $\mathbb{R}^{d}$, and therefore the state of the collection of $N$ particles is a point $(\bar{x}, \bar{v})$ in the $2 d N$-dimensional space $\left(B_{V}\right)^{N} \times \mathbb{R}^{d N} \subset \mathbb{R}^{d N} \times \mathbb{R}^{d N}$. The basic structure of statistical mechanics is a parametric family of probability distributions on the space of states of the many-particle system, the parameters being whatever thermodynamic quantities are needed to fully specify the large-scale behavior of the material. For simple materials there is a variety of alternative parameter pairs such as (pressure, temperature) or (temperature, mass density), and for each choice one has a corresponding 2-parameter family of probability distributions, called an ensemble. (Water freezes at $0^{\circ}$ Celsius at sea level, but this varies slightly with altitude, so temperature is clearly insufficient to specify the state.) Though they differ for small systems, for macroscopic systems the various ensembles are all fully equivalent, amounting to changes of variables affected by so-called Legendre transforms.

To define the pressure/temperature or $(P, T)$ ensemble for $N$ particles, we first confine the particle positions within the cubic box $B_{V}$ and give the configurations the relative probability density $e^{-E_{N} / T} d x d v$, where $E_{N}=$ $E_{N}(\bar{x}, \bar{v})$ is the total energy of the particles. (Note that the lower the temperature, the more the distribution is concentrated on small values of energy.) Then we sum or integrate over all volumes $V$ with a weighting factor $e^{-P V / T}$; high pressure concentrates the distribution on small volumes.

Putting this all together and normalizing the probability distribution, the average of a function $g$ of positions and velocities of the particles, for instance $E_{N}$, is given by

$$
\begin{aligned}
& \langle g\rangle_{P, T}=\frac{1}{Z_{N}(P, T)} \\
\times & \int_{0}^{\infty}\left[\int_{\left(B_{V}\right)^{N} \times \mathbb{R}^{d N}} g(\bar{x}, \bar{v}) e^{-E_{N}(\bar{x}, \bar{v}) / T} d \bar{x} d \bar{v}\right] e^{-P V / T} d V,
\end{aligned}
$$

where $Z_{N}(P, T)$ is the normalization constant or partition function:

(3)

$$
Z_{N}(P, T)=\int_{0}^{\infty}\left[\int_{\left(B_{V}\right)^{N} \times \mathbb{R}^{d N}} e^{-E_{N}(\bar{x}, \bar{v}) / T} d \bar{x} d \bar{v}\right] e^{-P V / T} d V .
$$

That's it. For dimension $d=3$ and sufficiently large $N$ this is the standard starting point for understanding everything we would like to know at the macroscopic level about matter in thermal equilibrium, from the relationship of the familiar solid, liquid, and gas phases in Figure 3 to properties such as the coefficient of thermal expansion as a function of temperature and pressure. For instance, the transitions in Figure 3 correspond to notable changes in the Gibbs free energy $G_{N}(P, T)=$ $(-T) \ln \left[Z_{N}(P, T) / N\right.$ ! $]$. Consequences obtained from this model are rarely straightforward or mathematically rigorous. It is nevertheless generally regarded as highly reliable by physicists because of a variety of nonrigorous evidence, including computer simulation and predictions verified by experiment. For the remainder of this article we will concentrate on the role that the box $B_{V}$ plays in the ensemble and will make free use of the nonrigorous intuition and results of this physical theory until a point where we can state some clean mathematical open problems.

We choose mass, time, and length units so that the total energy $E_{N}=E_{N}(\bar{x}, \bar{v})$ is the sum of the following kinetic and potential energies of the system:

$$
\begin{aligned}
& E_{N}^{k i n}=E_{N}^{k i n}(\bar{v})=\sum_{j=1}^{N} v_{j}^{2} / 2, \\
& E_{N}^{p o t}=E_{N}^{p o t}(\bar{x})=\sum_{j, k=1}^{N} \phi\left(\left|x_{j}-x_{k}\right|\right) .
\end{aligned}
$$




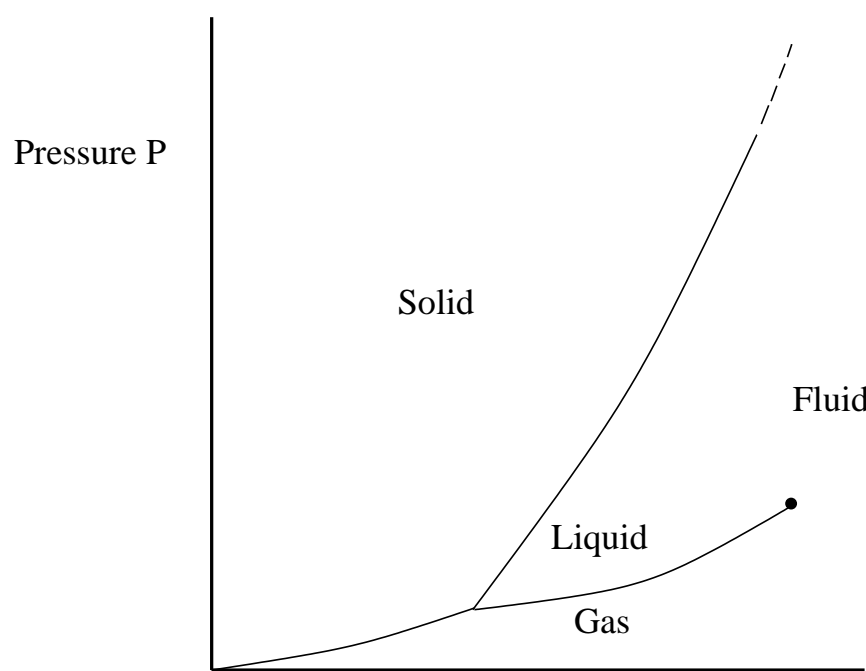

Temperature $\mathrm{T}$

Figure 3. Given an interaction potential $\phi$, the model determines a so-called phase diagram showing the separation between phases in terms of temperature and pressure.

Note that the only adjustable element of the model is the interaction (1), which is used in the potential energy $E_{N}^{p o t}(\bar{x})$, part of the total energy $E_{N}(\bar{x}, \bar{v})$, and that our choice of (1) makes sense in any dimension $d$. There turns out to be profound differences with dimension in the predictions of the model, which we discuss after introducing a new twist.

Recall that we have used a cube $B_{V}$ of volume $V$ as a container of the particles. We did not emphasize the shape because it has been proven that the predictions of bulk properties from such models, obtained from asymptotically large systems, are in fact independent of the shape of the container. But we are interested in the boundary shape of our water drop, which is not a bulk property, i.e. not proportional to $N$. With this in mind it will be useful to consider containers with a variety of shapes and introduce a new variable $S$, the fixed shape of our variable-volume container; we normalize $S$ to have unit volume. So now our $(P, T)$ ensemble also depends on $S$.

Trying to model the surface shape of a water drop adds another complication: the environment of the drop, oil in this case. Clearly the features of the water/oil contact are very relevant. If the oil is replaced by butyl alcohol, in which water dissolves to a noticable extent, the notion of interface is complicated by having a significant fraction of molecules of alcohol in the water and a significant fraction of water molecules in the alcohol. To get a simpler example we need something like motor oil, in which water is immiscible and which produces a very sharply defined interface between the water and oil. By modelling the oil as we have done the interface cannot affect the energy of the water molecules, except perhaps by the shape of the interface, which is very useful, as we

will see. We recall from (4) that the average total energy, $\left\langle E_{N}\right\rangle_{P, T}$, has both kinetic and potential portions:

$$
\left\langle E_{N}\right\rangle_{P, T}=\left\langle E_{N}^{k i n}\right\rangle_{P, T}+\left\langle E_{N}^{p o t}\right\rangle_{P, T},
$$

and from (2) we see that

$$
\left\langle E_{N}^{k i n}\right\rangle_{P, T}=\frac{\int_{0}^{\infty} E_{N}^{k i n}(\bar{v}) e^{-E_{N}^{k i n}(\bar{v}) / T} d \bar{v}}{\int_{0}^{\infty} e^{-E_{N}^{k i n}(\bar{v}) / T} d \bar{v}},
$$

which is not affected by the shape of the container. So to minimize the effect of the boundary the drop must attain that surface shape $S$ which minimizes the effect on the average potential energy, $\left\langle E_{N}^{p o t}\right\rangle_{P, T}$.

Intuitively, the presence of the boundary is felt directly only by those water molecules near the boundary; instead of being fully surrounded by water molecules, those near the boundary are "missing" some neighbors. From (1) each contribution to the potential energy is nonpositive, so the presence of a boundary increases the average potential energy, and minimizing

\section{confront a serious complication.}

its effect means choos ing that shape $S$ which minimizes $\left\langle E_{N}^{p o t}\right\rangle_{P, T}$.

Here we need to confront a serious complication. Minimizing $\left\langle E_{N}^{p o t}\right\rangle_{P, T}$ for finite $N$ might produce one or more rather complicated surfaces $S_{N}(P, T)$; what we want is the Wulff shape $S_{W}(P, T)$, the $\lim _{N \rightarrow \infty} S_{N}(P, T)$. As we will see, the latter may even be one or more accumulation points rather than a limit, but in any case in order to prove anything one must make some useful choice of a space of surfaces, with a topology on it in which we supposedly take our limits. A natural approach would be to limit ourselves to star-shaped surfaces containing unit volume, or even convex star-shaped surfaces, which are describable by a radial coordinate as a (piecewise smooth) function of angle (point on the unit sphere). And to reduce redundancy one could take the quotient of such surfaces by Euclidean congruence. Such matters are easy. It is much harder to choose a topology in which one can prove existence and uniqueness of our limits. Our optimization problem is entering the domain of the calculus of variations but with a quantity to minimize, $\left\langle E_{N}^{\text {pot }}\right\rangle_{P, T}$, which has the special probabilistic features of statistical mechanics. Such a calculus has not been worked out yet.

Ignoring that thorny "technical" issue, at least for now, we note that from the formula for $\left\langle E_{N}^{p o t}\right\rangle_{P, T}$ and from our expectation that for $(P, T)$ in the fluid phase our probability distribution will, for large $N$, be concentrated on highly disordered configurations of molecules, simple symmetry considerations should then imply that the limit of the minimum of $\left\langle E_{N}^{\text {pot }}\right\rangle_{P, T}$ is attained at that $S=S_{W}(P, T)$ which minimizes the surface area for fixed volume, namely the sphere. This is in satisfying agreement with the classical intuitive picture, which ignored molecules.

The more interesting situation occurs when $(P, T)$ is in the solid phase. We note from (2) that for fixed $P>0$, as $T$ 
decreases to 0 the $(P, T)$ ensemble becomes concentrated on those configurations $\bar{X}$ which minimize

$$
E_{N}^{p o t}(\bar{x})=\sum_{j, k=1}^{N} \phi\left(\left|x_{j}-x_{k}\right|\right),
$$

which is crystalline for our very simple (1). In more detail, if the boundary shape $S$ in our ensemble were a sphere, those potential crystalline configurations would be oriented equiprobably in every direction; the breaking of symmetry comes about by the minimization of $\left\langle E_{N}^{p o t}\right\rangle_{P, T}$ by a surface $S$ which is not rotationally symmetric.

It is expected that this effect is retained as $T$ increases from 0 until the point where the transition occurs to a fluid phase. The optimizing Wulff shape, $S_{W}(P, T)$, is presumably polyhedral in the solid phase, though none of this has been proven of course.

This is the standard picture in three dimensions: very pretty, indeed impressive, but no surprises for physicists. It does pose important challenges for mathematicians to justify all the "we expect"s, of course. But that is not the focus of this article. Rather, at this point we continue to draw a bit more from the nonrigorous theory by noting a significant difference in two dimensions from the Mermin-Wagner theorem, which proves that the solid state cannot be crystalline in two dimensions! In two dimensions it is not hard to show that the minimum of the potential energy from (1) is crystalline in the strict sense that the particles must sit on a triangular lattice; the Mermin-Wagner theorem shows that this orderly behavior is at least partly destroyed at any $P, T>0$. Symmetry principles have been useful in the standard physics of three-dimensional materials but cannot be as useful here. The 2016 physics Nobel award was given for developing a picture of phases in two-dimensional systems distinguished by topological features rather than by symmetry, but it is not clear that even this would help for our simple model of fluid and solid phases in two dimensions.

Going beyond the solid phase in two dimensions, there has been little analysis of what to expect for the more complicated notion of the optimal Wulff shape $S_{W}(P, T)$ for a finite piece of the system in the solid phase. Determining the Wulff shape or even determining the structure of solid configurations for our model is presumably very hard. But even without solving either of these one might be able to solve the following, which would still be of real interest:

Problem: Prove, in two dimensions, whether or not there is some region of $(P, T)$ values throughout which the optimal Wulff shape $S_{W}(P, T)$ is not the circle.

The circle is presumably the Wulff shape for sufficiently small $P$ and large $T$, so a positive solution to the problem would imply that one could "see" the effect of atomic structure in two-dimensional materials at a macroscopic scale, as in the facets of salt grains. And we note that a positive solution might be obtainable using nonoptimal shapes, avoiding the need to prove the existence of an optimal Wulff shape, "solid phase," or other nonrigorous terms we used as a guide.
We have shown how minimizing surface tension leads to interesting physics and mathematics when analyzed in two dimensions rather than the standard three dimensions. We conclude by noting a different nontraditional path concerning Wulff shapes that also leads to interesting mathematics, namely, if one chooses the parameters $(P, T)$ precisely on the phase transition curves in Figure 3. This is easy to understand in terms of our water drop suspended in oil. Assume fixed $P$ higher than the gas/liquid transition curve, and slowly decrease $T$, starting from large $T$, by pulling energy out of the water, i.e. by cooling it. When $T$ reaches the water/ice transition curve we start to change some water to ice, but the temperature does not actually change until all the water has become ice, and then the temperature starts to decrease again. The delicate situation where there is ice surrounded by water is reminiscent of our water drop surrounded by oil, but the conditions are subtly different. The ice/water interface is maintained by molecules moving back and forth between the water and the ice, equilibrium achieved by having the two back-and-forth rates being the same. This is very different from the water/oil interface, where we reasonably modelled the oil by an impenetrable but very flexible wall. Intuitively, the boundary between the ice and water is fuzzier, and it is harder to make sense of a "boundary" between the ice and the water.

A similar analysis can be performed for $(P, T)$ on the interface between water vapor and liquid water. This time the typical molecular configurations are isotropic in both phases, so the gas/liquid interface should still be spherical, but again it would be fuzzy and hard to make sense of, especially near the high temperature endpoint of the transition between gas and liquid in Figure 3. This situation has been analyzed quite carefully in a model where the particles can only live on the vertices of a square lattice in the plane. In that model, the lattice gas version of the so-called ferromagnetic Ising model, there is only a gas/liquid transition, so the goal is to clarify the interface between coexisting liquid and vapor in the presence of the fuzziness. Overcoming this complication, which we gladly ignored for the water/oil situation, was a tour-de-force in the probability subfield of limit shapes.

To summarize, we have considered minimization of surface tension for large collections of interacting molecules in two extreme cases: where the collection is in contact with a material with which it interacts minimally, such as water in contact with motor
There is an unlimited amount
of wonderful
mathematics in
such settings. oil, and where it is in one of two coexisting phases, such as liquid water in contact with water vapor, where there is significant interaction. This leaves out most of chemistry, where abutting materials can do all sorts of complicated things, such as iron rusting in air. There is an unlimited amount of wonderful mathematics in such 
settings, which is largely unexplored for its mathematics, especially in two dimensions, where even the traditional guide of symmetry is missing.

\section{References}

A. Okounkov, Limit shapes, real and imagined, Bull. Amer. Math. Soc. (N.S.) 53 (2016), 187-216. MR 3474306

D. RuELLE, Statistical Mechanics; Rigorous Results, Benjamin, New York, 1969. MR 0289084

J. E. TAYLOR, Crystalline variational methods, Proc. Nat. Acad. Sci. 99 (2002), 15277-15280. MR 1946763

\section{Previous Notices Articles by the Author}

Feature: A revolutionary material, March 2013.

Book Review: Quasicrystals and Geometry, by Marjorie Senechal, April 1996.

Feature: Symmetry and tilings, January 1995.

\section{Photo Credits}

Figure 1, Figure 3, and photo of author are courtesy of Charles Radin.

Figure 2 is courtesy of Maxim Bilovitskiy.

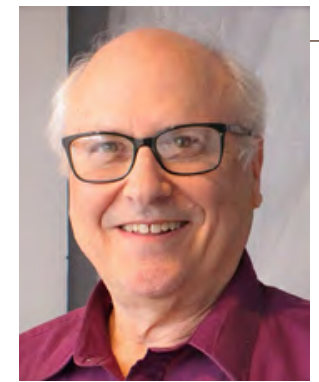

\section{ABOUT THE AUTHOR}

Charles Radin's training was in physics, and his research is in mathematical physics, in particular the study of the solid/fluid phase transition.
Charles Radin

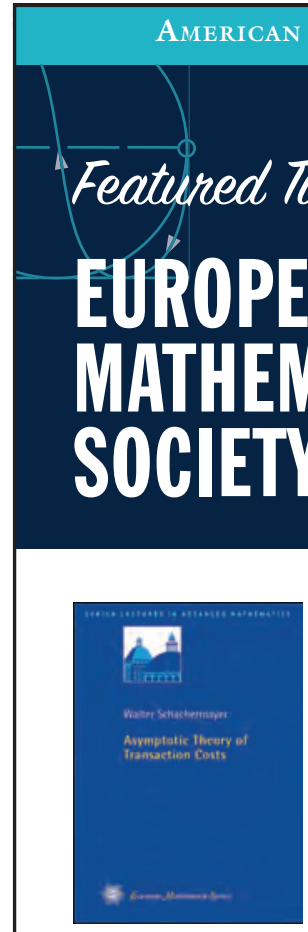

\section{Asymptotic Theory of Transaction Costs}

Walter Schachermayer, University of Vienna, Austria

A classical topic in mathematical finance is the theory of portfolio optimization. These lecture notes present some striking recent results of the asymptotic dependence of the relevant quantities when transaction costs tend to zero.

Zurich Lectures in Advanced Mathematics, Volume 23; 2017; 160 pages; Softcover; ISBN: 978-3-03719-173-6; List US\$38; AMS members US\$30.40; Order code EMSZLEC/23

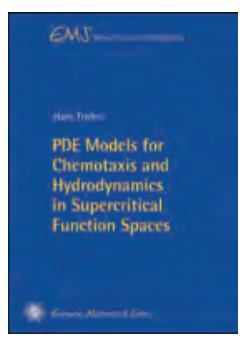

PDE Models for Chemotaxis and Hydrodynamics in Supercritical Function Spaces

Hans Triebel, University of Jena, Germany

This volume deals with PDE models for chemotaxis (the movement of biological cells or organisms in response to chemical gradients) and hydrodynamics (viscous, homogeneous, and incompressible fluid filling the entire space) paying special attention to mapping properties of related nonlinearities.

EMS Series of Lectures in Mathematics, Volume 27; 2017; 138 pages; Softcover; ISBN: 978-3-03719-172-9; List US\$38; AMS members US\$30.40; Order code EMSSERLEC/27

Publications of the European Mathematical Society (EMS). Distributed within the Americas by the American Mathematical Society.

Explore the complete collection of titles that we have to offer at bookstore.ams.org

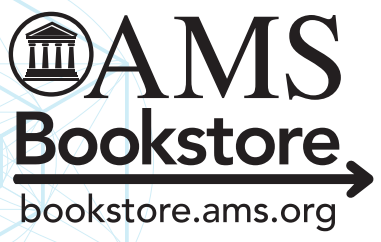

